$\begin{array}{ll}\text { Italique } & \text { Italique } \\ \text { Poésie italienne de la Renaissance }\end{array}$

V | 2002

Varia

\title{
Ludovico Castelvetro e Filippo Valentini in due sonetti di corrispondenza
}

Alberto Roncacci

\section{(2) OpenEdition}

Journals

\section{Edizione digitale}

URL: http://journals.openedition.org/italique/148

DOI: 10.4000/italique.148

ISSN: 1663-4438

\section{Editore}

Librairie Droz

\section{Edizione cartacea}

Data di pubblicazione: 31 dicembre 2002

Paginazione: 77-92

ISBN: 2-600-00826-8

ISSN: 1423-3983

Notizia bibliografica digitale

Alberto Roncacci, «Ludovico Castelvetro e Filippo Valentini in due sonetti di corrispondenza », Italique [Online], V | 2002, online dal 06 octobre 2009, consultato il 20 avril 2019. URL : http://

journals.openedition.org/italique/148 ; DOI : 10.4000/italique.148

(C) Tous droits réservés 
Alberto Roncaccia

L U D O V I C O C A S T E L V E T R O

E F I L I P O V A L E N T I N I

I N D U S ONETTI

D I C ORRIS P O N E N Z A 


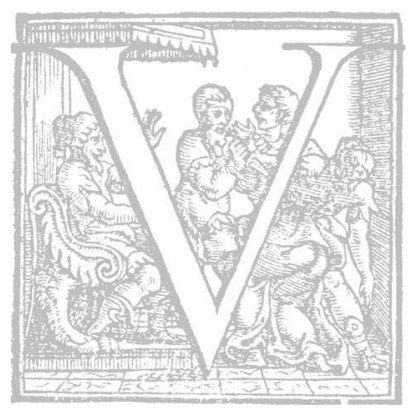

Na tra le testimonianze note della produzione volgare in versi riferibile all'Accademia modenese del Grillenzoni ${ }^{I}$ è costituita da due sonetti di corrispondenza scritti da Filippo Valentini ${ }^{2}$ e da Ludovico Castelvetro. L'interesse intrinseco dei due testi è senźaltro di tipo documentario, sia in senso biografico, sia in riferimento alla riflessione critica e alla produzione letteraria volgare contestualizzata all'ambiente culturale modenese a cavallo degli anni Trenta e Quaranta del Cinquecento. La fonte dei due testi è una pubblicazione settecentesca, larvatamente enciclopedica, ben nota agli studiosi del Castelvetro. Si tratta della Raccolta d'opuscoli scientifici e filologici, curata da Angelo Calogerà e stampata in 5 I volumi a Venezia, presso Simone Occhi, tra il 1728 e il 1754. Il Calogerà poté avere accesso, tra l'altro, a documenti oggi non più disponibili, appartenenti a collezioni private, come fu il caso del manoscritto da cui trasse $i$ due sonetti che ci interessano e di cui egli riferisce, con tutta probabilità, l'epigrafe originale: «Rime manuscritte di M. Filippo Valentini»». ${ }^{3}$ Di questi due sonetti, esemplati da quel codice per interesse relativo al solo Castelvetro, quella stampa fornisce l'unico testimone, cui hanno in seguito fatto riferimento le due uniche citazioni-riedizioni successive, una parziale del primo Novecento, all'interno di una monografia sul Castelvetro, ed una recentissima, all'interno di un lavoro monografico sul Valentini. Nel primo caso si tratta dell'ancora importante studio di Giuseppe Cavazzuti, ${ }^{4}$ molto ricco di riferimenti bibliografici e interessante anche per la segnalazione di interventi poco ortodossi del Muratori su manoscritti castelvetriani posseduti dalla Biblioteca Estense di Modena. Il secondo studio è l'edizione, curata da Lucia Felici, del noto trattato pedagogico che il Valentini dedicò ad Ercole II e a Renata di Francia, importante per l'introduzione che costituisce, come si è detto, una vera e propria monografia su questo autore. Nessuno di questi due studi, che pur integrano i testi, a scopo esemplificativo, nel loro discorso, ne offre tuttavia una lettura puntuale. Bisogna precisare che il Cavazzuti cita il solo sonetto del Castelvetro, cosicché la corrispondenza è per la prima volta accessibile al lettore moderno solo all'interno del citato saggio della Felici. Se il Cavazzuti ripubblica il sonetto del Castelvetro fornendo una punteggiatura interpretativa soddisfacente per il lettore moderno, la Felici tende invece a conservare la quasi totalità dell'interpunzione calogeriana, con tutta probabilità ripresa meccanicamente dall'originale, ed offre una sorta di trascrizione semi-diplomatica che accresce, più che diminuire, l'oscurità complessiva dei due sonetti, cosi da risultare ecdoticamente improponibile.

Mi sembra perciò opportuno fornire la trascrizione diplomatica della stampa settecentesca. I due testi, che si trovano rispettivamente alle pp. 95 e 96 del tomo 
XXXVII della Raccolta calogeriana, sono preceduti, alla fine di p. 94, dalla didascalia che ugualmente si trascrive:

\section{[p. 94]}

I seguenti due Sonetti, non più stampa- / ti, si trovano fra le Rime Manuscritte / di M. Filippo Valentini, che fu gran- / de amico del Castelvetro. // SO.

[p. 95]

SONETTO / Di M. / LODOVICO CASTELVETRI / A M. / PHILIPPO VALENTINI. / PROPOSTA, // Se non vedesti ancor per lunghe o torte / Vie da l'usato corso suo smarrita / Punto la queta mente mia romita, / A che pur spargi al ciel parole morte. // Se sottilmente la strema mia sorte, / Come ben scorgi, a sostentar m'aita, / Et o non manchi innanzi la partita, / Ch'io lasci il troppo a che pur mi co[n]forte. // Chi non sa ch'al varcar di questa valle / Di lagrime, la qual ogni or menzogna, / Spirto rabbioso turba d'alto a valle; // Chi non vuol affogarvi con vergogna / Fa mestier c'abbia intorno molte galle; / Dunque il consiglio tuo par d'huom, che sogna. // RI.

\section{[p. 96]}

RISPOSTA. // Et larga strada, \& ampia entrata a morte / Altrui condurre agiato, \& carco in vlta, / Ma disaggioso, \& scarco a la vita / Per angusto sentier povere porte. // Chi quà giù scese a far le genti accorte / Con opre \& con parole ognior n'addita, / U' non d'agi, \& di some alma impedita / Entrar più che per ago le ritorte. // A si picciola porta, \& stretto calle / Tutti i comodi uman lasciar bisogna, / Et d'ogni peso disgombrar le spalle, // Che passarvi altrimente in van s'agogna, / Se 'l saver di colui forse non falle, / Cui senza, Atene san nulla, e Bologna.

Si può osservare, in prima approssimazione, come $i$ due sonetti debbano supporre una corrispondenza conclusa, visto che il Calogerà, interessato a pubblicare testi inediti o rari del Castelvetro, avrebbe di certo riportato altri eventuali sonetti di lui che fossero inclusi nel manoscritto delle Rime del Valentini. Ad una lettura che tenga conto del senso logico complessivo dei due componimenti non può sfuggire come la sequenza vada invertita. La conclusione del sonetto del Castelvetro denota infatti il senso di una risposta data ad un «consiglio» ricevuto, "consiglio» che puntualmente troviamo nella prima terzina del sonetto del Valentini: "Tutti i comodi uman lasciar bisogna, / Et d'ogni peso disgombrar le spalle». Ė quanto d'altra parte sembra aver inconsapevolmente intuito a suo tempo il Cavazzuti, il quale nell'introdurre il sonetto del Castelvetro dice, senza tener conto del fatto che la trascrizione calogeriana lo presenta come proposta, "Risponde al Valentini»." 
Restaurato l'ordine di successione, regolarizzata la grafia ed aggiunta un'interpunzione di tipo interpretativo, ecco come si presentano $i$ due sonetti:

Proposta di Filippo Valentini

Et larga strada et ampia entrata a morte

Altrui condurre agiato et carco invita,

$\mathrm{Ma}$, disaggioso et scarco, a la vita

Per angusto sentier, povere porte

Chi qua giù scese a far le genti accorte

Con opre et con parole ognior n'addita,

U' non d'agi et di some alma impedita

Entrar più che per ago le ritorte.

A sì picciola porta et stretto calle

Tutti i comodi uman lasciar bisogna

Et d'ogni peso disgombrar le spalle,

Ché passarvi altrimente in van s'agogna,

Se 'l saver di colui forse non falle

Cui senza Atene san nulla e Bologna.

Risposta di Ludovico Calstelvetro

Se non vedesti ancor per lunghe o torte

Vie da l'usato corso suo smarrita

Punto la queta mente mia romita,

A che pur spargi al ciel parole morte?

Se sottilmente la strema mia sorte,

Come ben scorgi, a sostentar m'aita

(Et oh non manchi innanzi la partita!)

Ch'io lasci il troppo a che pur mi conforte?

Chi non sa ch'al varcar di questa valle

Di lagrime, la qual ognior menzogna,

Spirto rabbioso, turba d'alto a valle,

Chi non vuol affogarvi con vergogna

Fa mestier ch'abbia intorno molte galle?

Dunque il consiglio tuo par d'huom che sogna.

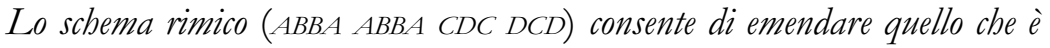
forse soprattutto un refuso tipografico, situato alla fine del secondo verso del sonetto del Valentini, dove il testimone a stampa legge "carco in vlta». La Felici interviene e legge "carco in ulta», a scapito di rima e di senso. Si tratta invece di una piu comprensibile confusione di «i emendare, a rigor di senso logico e di rima, con «carco invita» 
Per quanto riguarda l'interpretazione dei due testi, lo studio della Felici, che come si è detto mantiene l'erroneo ordine di successione del testimone a stampa, ci offre alcune indicazioni di tipo biografico e contenutistico. Rilevata la centralità della tematica religiosa, la studiosa osserva come il Valentini rivendichi, nei confronti del Castelvetro, «la propria impegnativa, ma irrinunciabile scelta di imboccare la strada angusta indicata da Cristo, come la sola in grado di condurre alla verità», ed ipotizza, inoltre, come tale scelta non fosse "genericamente quella di seguire l'Evangelo, ma bensi quella, assai più radicale, di accostarsi alle dottrine valdesiane». ${ }^{6}$ In proposito, e molto opportunamente data la prossimità tematica, la Felici trascrive dal Tiraboschi il seguente brano di una lettera del Castelvetro indirizzata al Valentini nel I537 (sottolineature mie):

Io se vi debbo dire il vero, quanto ho compreso dalle vostre parole, queste vie non calpestate non mi paiono della semplice dottrina di Cristo, ma piuttosto Chimere, e dimostrazioni piuttosto di un ingegno poetico che cristiano. Che catene, che anella son queste che mi scrivete? O che questi secreti non si possono sapere che Dio ce gli ha nascosti, o che le vie piane e frequentate dall'Evangelio sono buone a menarci ad essi. ${ }^{7}$

Restando a livello puramente testuale, è possibile confermare, in ogni modo, solo l'evangelismo dell'invito a seguire alla lettera l'esempio della povertà di Cristo. L'importanza della lettera citata è un'altra, quella di fornirci, data la corrispondenza non solo del tema trattato, ma anche della precisa opposizione vie non calpestate / vie piane e frequentate, analogamente presente nei sonetti, uno strumento di datazione relativamente soddisfacente. I due testi sarebbero quindi stati composti intorno al I537, cioè nel periodo in cui, secondo la corrispondenza citata, Castelvetro e Valentini si confrontarono sullo stesso tema evangelico espresso nei sonetti.

Prima di considerare alcuni elementi più propriamente formali, propongo a verifica del senso letterale la seguente parafrasi.

\section{Proposta del Valentini:}

Una strada larga e un'ampia entrata conducono chi vive negli agi e nella ricchezza verso la morte spirituale. Ma Colui che, senza comodi e poveramente, venne in Terra per rendere avveduti gli uomini attraverso opere e parole, sempre ci addita per un impervio cammino la angusta porta ${ }^{8}$ della vita eterna, attraverso la quale l'anima non impedita da agi e some terrene può entrare più facilmente che una fune per la cruna di un ago. ${ }^{9}$ Per oltrepassare una così piccola porta e un così stretto cammino bisogna lasciare tutti i comodi terreni e sgombrare d'ogni peso le spalle, perché altrimenti invano si desidera di passarvi, se non erra il sapere di Colui senza il quale non è nulla il sapere di Atene e di Bologna. 
Risposta del Castelvetro:

Se ancora non hai veduto la mia mente quieta e solitaria affatto smarrita dal suo usuale cammino per vie lunghe e tortuose, perché allora pronunci parole che, alla prova dei fatti, si rivelano vuote e inconsistenti? Se la sorte dei miei ultimi anni mi aiuta, come ben vedi, a vivere in semplicità (e voglia il cielo che ciò non venga a mancare prima della morte!), perché allora mi inviti a lasciare il troppo? Chi non sa che al passaggio di questo mondo - il quale dall'inizio alla fine è sempre turbato dallo spirito rabbioso della menzogna - è necessario che chi non voglia esser travolto con vergogna abbia intorno a sé molti sostegni? Il tuo consiglio è dunque degno d'uomo che vaneggia.

La parafrasi mostra, in sostanza, come Castelvetro restando su posizioni che la Felici ben definisce "razionali e bibliche», che è come dire più moderatamente erasmiane o umanistiche, rifiuti le sollecitazioni esplicitamente evangeliche del Valentini. Contro tale moderatismo sembra da intendere l'ultimo verso del sonetto del Valentini, in cui le due città potrebbero rappresentare metonimicamente il sapere umanistico e il sapere giuridico caratteristici del Castelvetro, il quale oltre che grecista, ovvero umanista completo, aveva completato gli studi di diritto a Bologna (come d'altra parte il Valentini stesso). ${ }^{\text {Iо }}$ Ora, da un punto di vista formale, si può notare come la fisionomia stilistica del primo sonetto resti tutto sommato all'interno dei codici lessicali, sintattici e metrici del petrarchismo cinquecentesco, seppure, direi, in posizione liminare, fosse solo per il fatto di proporre un nucleo di riflessione civile-religiosa non indolore, soprattutto considerati $i$ tempi. Una certa presa di distanza dall'ortodossia bembiana è comunque rilevabile in forme non consacrate come «disaggioso» ( $v .3)$, come il dantesco o comunque comico «ritorte», ${ }^{\mathrm{I}}$ o come il decisamente dantesco «s'agogna». ${ }^{2}$ S'aggiunga inoltre una certa disponibilità all'accento tonico di settima posizione, rilevabile nel $v$. 3 (si noti la dialefe «disaggioso et»), e nel v. I4, dove è in particolare risalto per il fatto di corrispondere ad uno dei concetti chiave del sonetto e perché situato in verso conclusivo (disponibilità sottoscritta dal Castelvetro nel quinto verso del suo sonetto). ${ }^{13}$ Si tratta di piccole incrinature sonore e lessicali, certo, ma che lasciano intuire una certa discrepanza dalle proposte teoriche del Bembo, al di là degli indubbi rapporti di amicizia che il Valentini ebbe con il Veneziano. ${ }^{\mathrm{I}}$ Una decisa aspettativa in senso progettualmente antibembiano viene del resto testimoniata, nei confronti del Valentini, dallo stesso Castelvetro in una nota lettera, datata ancora una volta I537. ${ }^{\text {IS }}$ Studi specifici sul Valentini poeta potranno forse confermare queste impressioni, tenuto conto che in tale direzione sembra andare la pratica di un metro lungo come la canzone, ${ }^{\mathrm{I}}$ d'argomento per di più civile, ad un'altezza cronologica in cui s'erano ormai consolidati, in coincidenza, quelli che Dionisotti definiva «trionfo del sonetto» e 
«precipitoso declino della canzone»». ${ }^{17}$ Valentini, in controtendenza, emula virtuosisticamente il Petrarca civile di Italia mia, benché 'l parlar sia indarno, riprendendone lo schema metrico e componendo addirittura venticinque stanze nella canzone Poi perch'amor per donna senza pare, incentrata tematicamente sull'esaltazione dell'opera unificatrice di Carlo $V^{18}$

Nella detta direzione antibembiana, ma con indicatori stilistici più radicali e sicuri, si muovono le scelte formali del sonetto del Castelvetro. Si tratta di scelte su cui risulta pesare - irreparabilmente, si è tentati di dire - soprattutto il pensiero del critico e del commentatore sperimentato, che non la consuetudine e la dimestichezza operativa del comporre in versi. Sono, tuttavia, proprio questo tipo di evenienze che si intendono analizzare. In proposito, il Cavazzuti non poté esimersi da esplicite ironie, infastidito in particolare dall'ultima teriina, osservando:

Immaginate una valle gonfia di lagrime e l'uomo fortunato che passa nuotando, sorretto da molte galle? e avete mai trovato nel Petrarca un verso simile: Fa mestier c'abbia intorno molte galle? Buon Castelvetro! lasciamolo in pace: perché 'uccidere un uomo morto'? ${ }^{\text {I9 }}$

Il Cavazzuti ha ragione nel notare che il v. Iz è lontanissimo da Petrarca, ma paradossalmente indica proprio la componente piu interessante del componimento, quella della trasgressione, più che nei confronti di Petrarca, si potrebbe dire, in quelli di una certa maniera di riferirsi a Petrarca. E si può precisare subito che tale trasgressione consiste in una serie di rinvii metatestuali ad un altro modello privilegiato del Modenese, cioè a Dante, non estraneo, come si è visto, neppure al precedente sonetto del Valentini. Non è qui il caso si soffermarsi sul grado di riuscita stilistica del sonetto. Interessa invece determinare quale tipo di operazione critica, di riflessione letteraria, consenta al Castelvetro di pretendere coerenza ed efficacia per un'operazione di contaminazione cosi poco convenzionale per l'epoca sua e, in verità, ancora per la nostra. ${ }^{20}$

Il contesto di produ₹ione del sonetto, specificamente modenese, e le alte competenze letterarie del destinatario, fanno si che si possa supporre da parte del Castelvetro un investimento elevato e consapevole di risorse formali. S'aggiunga che per quanto riguarda il tema del distacco dalle cose mondane, a spiegare $i$ due testi potrebbe bastare l'esercizio di ripresa di precedenti tematiche petrarchesche. Avremmo in questo senso un riferimento al Petrarca più ragionativo, ad esempio, delle canzoni d'argomento civile-religioso. Vari elementi stilematici e metrici possono essere contrassegno di richiami tematici. In particolare, possiamo far riferimento alla prima strofa della canzone petrarchesca $\mathrm{O}$ aspectata in ciel beata e bella (Rvf. 28), al sonetto Poi che voi 
et io più volte abbiam provato (Rvf. 99), o all'ultima strofa di Italia mia, benché 'l parlar sia indarno (Rvf. I28). La «valle / di lagrime» della prima terzina, al di là dell'evidente richiamo alla tradizione liturgica, ${ }^{21}$ è evocata da Petrarca nelle due canzoni appena citate: «questa oscura valle, / ove piangiamo» (Rvf. 28, II-I2), «Al passar questa valle» (Rvf. I28, I03). Si noti come il settenario appena citato sia riecheggiato puntualmente da Castelvetro anche grazie all'enjambement: "ral varcar di questa valle / di lagrime». Dal sonetto 99 si possono citare per intero le due terzine: "Voi dunque, se cercate aver la mente / anzi l'extremo di queta già mai / seguite $i$ pochi, et non la volgar gente. / Ben si può dire a me: Frate, tu vai / mostrando altrui la via, dove sovente / fosti smarrito, et or se' più che maì. Ritroviamo in questi versi il sintagma «queta mente» del sonetto di Castelvetro $(v .2)$, con inoltre la coincidenza tematica tra espressioni come «anzi l'extremo dì e «innanzi la partita» (v. 7). Lessicalmente vanno segnalate le presenze di «extremo» (Rvf. 99, Io) per «strema» (v. 5) e di «smarrito» (Rvf. 99, I4) per «smarrita» (v.2).

Grazie a tali rinvii tematici a Petrarca, per $i$ due sonetti non è strettamente necessaria un'interpretazione in termini autobiografici. In chiave di imitazione petrarchesca si rileva la scelta di una tematica diversa da quella più fortunata dell'amore infelice, volta a secondare, si può aggiungere, una maggiore capacità di accoglimento lessicale e sintattico-ritmico. In questo senso, l'espressione castelvetriana «queta mente» rinvia ad un sintagma petrarchesco che realizza, tecnicamente, un uso raro nel Canzoniere, quello dell'enjambement concernente la coppia aggettivo-sostantivo, o, come preferi dire Contini, epiteto-sostantivo, ${ }^{22}$ e costituisce quindi l'esempio di una infrazione, soprattutto quando $i$ due versi in tmesi siano endecasillabi, ${ }^{23}$ alla tendenziale unità logico-sintattica del verso. Lo stesso tipo di tmesi ritmica è infatti collocato da Castelvetro in posizione di prima evidenza nel passaggio tra primo e secondo verso: "per lunghe o torte / vie». Si tratta anche in questo caso del recupero di uno stilema petrarchesco, la cui memoria è sottolineata dalla conservazione della rima -ORTE: "giunto il vedrai per vie lunghe e distorte» (Rvf. 37, 24). In questo caso, a differenza del precedente in cui il sintagma originariamente in enjambement viene ricomposto, la tmesi aggettivo-sostantivo è introdotta dal Castelvetro. Ė come se l'autore, dopo aver introdotto un enjambement all'apparenza non autorizzato dal punto di vista dell'usuale percezione del modello ("torte / vie»), fornisse all'orecchio esercitato del lettore contemporaneo il rinvio intertestuale per reperirne proprio la legittimazione petrarchesca («la queta mente»), rinvio che doveva costituire per $i$ contemporanei una sorta di esempio da manuale, data la singolarità del compimento dell'enjambement «mente / [...] queta» solo nel secondo emistichio del verso. Tale legittimazione tende ad investire anche il complessivo 
assetto formale della poesia, marcato da una sottolineatura di forte drammaticità data dalle scelte rimiche piuttosto aspre e dai forti enjambement dei vv. I-2, 2-3, e 9-I0. S'aggiunga, per sottolineare maggiormente il significato del sintagma «queta mente», che l'esito monottongato di queto e derivati è sistematico in Petrarca, mentre l'oscillazione con la forma dittongata è comune, ad esempio, nella poesia di Dante e Boccaccio.

Il recupero di uno stilema propriamente petrarchesco è ancora ravvisabile nel secondo emistichio del verso 5, «la strema mia sorte», che richiama Rvf. 298, Io «ch' $i$ ' porto invidia ad ogni extrema sorte». Si può in questo caso notare, in assenza di ragioni metriche, la preferenza del Castelvetro per la forma aferetica stremo, comune in poesia ma sistematicamente evitata dal Petrarca e, di conseguenza, dal Bembo delle Rime.

La volontà di evocazione del modello è, in ogni modo, sottolineata dal fatto che in posizioni fortemente marcate, quali sono quelle rimiche del verso iniziale e del verso finale del componimento, sono collocati due ulteriori stilemi petrarcheschi. Oltre il già citato «per vie lunghe e distorte» di Rvf. 37, 24 recuperato in «per lunghe o torte / vie», la chiusura del sonetto, "par d'buom che sogna», riprende due occorrenze e corrispondenze rimiche petrarchesche: «et quasi d'uom che sogna» (Rvf. 49, 8), "Ché 'n guisa d'uom che sogna» (Rvf. 264, 88). ${ }^{24}$ Il sonetto è, per cosi dire, aperto e chiuso, quasi con ostentazione, all'insegna di Petrarca. Tuttavia, all'interno di questa sorta di inquadratura petrarchesca, il risultato complessivo è ben poco intonato all'equilibrio sintattico e alla scelta lessicale propria dell'unilinguismo del Canzoniere. Sarebbe certo fare un torto all'autore, d'altra parte, negargli la capacità di versificare in maniera più consona al riuso del modello operata con facilità dalla maggior parte dei suoi contemporanei. Sembra quasi, piuttosto, che il modello sia evocato, se non per essere contraddetto, per essere contaminato, o comunque ripercorso liberamente; al punto, si potrebbe dire, di annullare l'intonazione petrarchistica che il ricorso a certi stilemi avrebbe invece potuto favorire.

Possiamo notare, per il verso messo alla berlina dal Cavazzuti, come l'espressione far mestiere, non sia mai usata in versi né da Petrarca, né da Bembo, ma sia molto comune in Dante. Si può citare, tra i tanti possibili, l'esempio "A costui fa mestieri, e nol vi dice» (Par. I4, I0). Lo stesso vale per "rgalle», di cui nella Commedia abbiamo diverse occorrenze rimiche. In un caso abbiamo la stessa rima del sostantivo plurale: "Tra brutti porci, più degni di galle» (Purg. I4, 43). In altri due casi abbiamo l'uso coningato del denominale gallare: «fanno attuffare in mezzo la caldaia / la carne con li uncin, perché non galli» (Inf. 21, 57), "Di che l'animo vostro in alto galla» (Purg. I0, I27). Quest'ultimo esempio, ̀̀ importante osservare, autorizza l'uso figurato dell'espressione in un contesto di senso elevato, quale è quello operato da Castelvetro. 
Il rinvio a Dante, oltre che determinato da riprese lessicali o rimiche, è anche contenutistico, alla pari di quello a Petrarca. Sorge infatti spontaneo nel lettore il richiamo allo smarrimento terreno del primo canto della Commedia. Si tratta di un rimando, tuttavia, non generico, ma misurabile su elementi ben precisi. La via smarrita di Dante è evocata dalla voce «smarrita» del v. 2 del sonetto, che all'altra estremità del verso è appunto abbinata metricamente alla voce «vie». Non senza significato è poi l'associazione per opposizione diritta via / torte vie. Dal punto di vista rimico, inoltre, anche le due uscite in -ITA $e$-ORTE rinviano ai primi versi del primo canto di Inferno. Lo stesso vale per «valle», che troviamo in rima al $v$. I4 dello stesso canto. Castelvetro la pone in rima, oltre che con «galle», con un altra espressione d'uso dantesco, cioè con l'avverbiale «a valle» (Inf. I2, 46), ${ }^{25}$ non usata da Petrarca. Anche la triade rimica menzogna-vergogna-sogna si può come tale considerare dantesca (Inf. 16, I22-26), oltre che petrarchesca (Rvf. 49).

Per gli elementi considerati, dato il tema topico dello smarrimento terreno (già almeno dantesco e petrarchesco), unitamente all'assenza di riferimenti biografici precisi, possiamo ribadire in linea generale come l'interesse specifico del sonetto non sia quello biografico. L'interesse, per gli echi testuali rilevati, è piuttosto formale e stilistico. Da tale punto di vista, nel contesto del diffuso petrarchismo amoroso cinquecentesco, caratterizzato da scelte lessicali e metriche tendenti ad evitare asprezze e scarti di tipo sia lessicale che sintatticoritmico, il sonetto può essere letto come espressione di una riflessione critica volta a rivalutare una pratica del rimare fatta invece di contenuti esistenziali, civili e religiosi, di possibilità lessicali e ritmiche molto ampie. Vi sarebbe affermato, implicitamente, il non necessario rispetto dei principi di gravità $e$ piacevolezza per $i$ quali Bembo riconosceva esemplare maestro Petrarca, come è noto, ma non Dante e Cino. ${ }^{26}$ Il giudizio negativo di Bembo su Dante, definito grave ma non piacevole, sembra trovare un forte correttivo in Castelvetro, se non un'inversione di segno del giudizio critico. Ne è prova il fatto che $i$ numerosi richiami danteschi siano per cosi dire incorniciati da due evidenti citazioni petrarchesche. Si può comprendere, insomma, come il modello petrarchesco proposto da Bembo potesse in realtà risultare limitativo nei confronti dello stesso Petrarca. In tal senso, il Castelvetro, cui va in questo con tutta probabilità accomunato il Valentini, non intendeva trascurarne la componente civile e religiosa, recuperata formalmente anche attraverso la trasgressione a quel principio di piacevolezza cui rispondono diffusamente l'unità metrico sintattica dei versi e la selezione lessicale.

La validità più generale di quanto osservato del sonetto può, inoltre, esser corroborata proprio dal fatto che esso sia indirizzato al Valentini, cioè all'altro principale ideologo letterario dell'Accademia, al quale del resto il Castelvetro fa esplicito riferimento in sede di commento a Petrarca. ${ }^{27}$ Lo 
scambio di sonetti tra $i$ due letterati lascia perciò verosimilmente presupporre, per $i$ reciproci interessi teorici, una implicita riflessione critica sulla scrittura volgare in versi. Risulterebbe percio fortemente limitativo considerare certi elementi contenutistici e formali solo all'insegna di una ingenua spontaneità autobiografica, come se fosse secondario il fatto di avere a che fare con una precisa costruzione in versi, ovvero con quella che potremmo definire una particolare, consapevole o meno, procedura di selezione dei codici.

Ci si può accontentare, provvisoriamente, di aver rilevato alcune direzioni di tale procedura nei due sonetti esaminati e soprattutto nel secondo. In prospettiva di ulteriori ricerche, si può comunque riflettere sul fatto che tale recupero di un certo plurilinguismo dantesco, se trasgredisce $i$ principi di gravità e di piacevolezza, già ciceroniani, prescritti dal Bembo in sede teorica, è paradossalmente in sintonia con un settore determinato delle scelte concrete operate dal Bembo poeta. ${ }^{28}$ Si pensi ad esempio alle osservazioni di Dionisotti sul Bembo della canzone Alma cortese, contrassegnata da indubbi dantismi lessicali e metrici, interpretati come «segno di un graduale calcolato e faticoso processo di liberazione dal modello [petrarchesco] verso uno spazio nuovo e più aperto». ${ }^{29}$ Tale componimento, che emula in senso sperimentale soprattutto la gravità e la testura arcaicizzante di una canzone molto antica nella storia interna del Canzoniere, qual è Nel dolce tempo de la prima etade, costituisce senz'altro un caso isolato, anche per livello di risultati, nella produzione lirica bembiana. ${ }^{30}$ Ad essa, e alla fase di poetica che implicitamente rappresenta, sembra non casualmente rinviare un altra piu cospicua corrispondenza in versi dell' Accademia modenese, in cui i primi due sonetti (su otto di vari autori che si rispondono per parole-rima) ne riecheggiano l'incipit. ${ }^{\text {I }}$ A tale direzione del petrarchismo cinquecentesco, non secondaria, anche se non vincente, ancora rappresentata dal Bembo dell'edizione Is30 delle Rime, ${ }^{32}$ crediamo percio che possano corrispondere, con consapevolezza propositiva, $i$ due sonetti del Castelvetro e del Valentini.

Alberto Roncaccia 


\section{Ludovico Castelvetro e Filippo Valentini}

Queste pagine nascono da una presentazione seminariale avvenuta nell'anno accademico 200I-O2 nell'ambito dell'Ecole doctorale d'Etudes italiennes delle Università di Ginevra e di Losanna.

I. Tra le pubblicazioni più recenti sull'Accademia modenese, si vedano S. Peyronel Rambaldi, Speranze e crisi nel Cinquecento modenese. Tensioni religiose e vita cittadina ai tempi di Giovanni Morone, Milano, Angeli, 1979; M. Firpo, Gli "spirituali", l'Accademia di Modena e il formulario di fede del Is42: controllo del dissenso religioso e nicodemismo, "Rivista di storia e letteratura religiosa», 20 (1984), pp. 40-I I I (ora in Inquisizione romana e Controriforma. Studi sul cardinal Giovanni Morone e il suo processo d'eresia, Bologna, Il Mulino, 1992, pp. 29- I I 8); P. Zambelli, 'Aristotelismo eclettico' o polemiche clandestine? Immortalità dell'anima e vicissitudini della storia universale in Pomponaz:i, Nifo e Tiberio Russiliano, in Die Pbilosopbie im I4. und Is. Jabrbundert. In memoriam Konstanty Michalski, Amsterdam, Grüner, 1988, pp. 535-72; M. Firpo - D. Marcatto, Il processo inquisitoriale del cardinal Giovanni Morone, Edizione critica, 6 voll., Roma, Istituto Storico Italiano per l'Età moderna e contemporanea, I981-95. Fondamentale resta comunque il riferimento a Girolamo Tiraboschi, Biblioteca modenese, Modena, Società tipografica, 178I-86, 6 voll., in part. vol. I, p. Iz e ss.

2. Per la biografia del Valentini si veda l'Introduzione di L. Felici a Filippo Valentini, Il principe fanciullo. Trattato inedito dedicato a Renata ed Ercole II d'Este, Firenze, Olschki, 2000, p. 5 nota Io.

3. Angelo Calogerì, Raccolta d'opuscoli scientifici e filologici, Venezia, presso Simone Occhi, vol. xxxvir (1747), p. 94. Nella Premessa il Calogerà, pur senza riferirsi al manoscritto di Rime del Valentini, riferiva di aver avuto a disposizione documenti di proprietà di Domenico Vandelli (p. 4). Probabilmente lo stesso manoscritto di Rime fu consultato in seguito anche dal Tiraboschi, che ci informa più precisamente, elencando le opere del Valentini, sul numero di componimenti: «Sonetti e Canzoni. Sono in numero di circa centosessanta in un codice della Libreria Vandelli» (Tiraboschi, Biblioteca modenese, vol. v, p. 3 I9). Per testimonianza del Tiraboschi sappiamo che tale codice conteneva altre cose, come la lettera in cui Bembo ringrazia il Valentini per un sonetto dedicatogli (p. 310).

4. G. Cavazzuti, Ludovico Castelvetro, Modena, Società Tipografica Modenese, 1903.

5. Cavazzuti, Ludovico Castelvetro cit., p. I96.

6. Felici in Valentini, Il principe fanciullo, p. 35.

7. Tiraboschi, Biblioteca modenese, vol. I, p. 485; poi citato dalla Felici in Valentini, Il principe fanciullo, p. 35.

8. Cfr. la sentenza evangelica in Luca I3, 23-24.

9. Cfr. Luca I 8,25 .

Io. Si confronti quanto scrive in merito la Felici in Valentini, Il principe fanciullo, p. I6.

i i. Cfr. Dante Alighieri, Inf. 19, 27 e Inf. 3 i, i i i; Cecco Angiolieri, Rime 88, 10.

I 2. Se ne vedano le occorrenze in Dante Alighieri, Inf. 6. 28; Inf. 26, 9; Inf. 30, I $38 ;$ Purg. I3, 66.

I 3. Tale elemento metrico, per il fatto di ripetersi in entrambi i sonetti, costituisce una coincidenza significativa. Lo schema dattilico costruito su accenti tonici di $4^{\mathrm{a}}$ e di $7^{\mathrm{a}}$, 
ancora quantitativamente consistente nel Canzoniere, risulta drasticamente ridotto nella produzione lirica bembiana, così da lasciar ragionevolmente supporre, per i due sonetti modenesi, un posizionamento autonomo nei confronti del modello petrarchesco (per il confronto di tale modulo ritmico in Petrarca e Bembo mi baso su M. Praloran, Metrica e tecnica del verso, in 'Prose della volgar lingua' di Pietro Bembo, Atti del convegno di Gargnano del Garda (4-7 ottobre 2000), a c. di S. Morgana, M. Piotti e M. Prada, Milano, Cisalpino, 2000, pp. 409-2I).

I4. Una attitudine critica nei confronti del Bembo viene colta, giustamente, dalla Felici, che però attribuisce erroneamente al Valentini le frammentarie Annotationi sopra $i$ sonetti del Bembo conservate presso la Biblioteca Reale di Copenaghen sotto la segnatura Gl. klg. S. 2057 , ff. I-6, che sono invece da ascrivere al Castelvetro (cfr. Felici in Valentini, Il principe fanciullo, pp. 37-38). Per la questione attributiva si veda, a cura di chi scrive, Un frammento critico sulle Rime del Bembo attribuibile a Ludovico Castelvetro, "Aevum», LXXIII (1999), pp. 707-33.

I 5. Tale lettera si legge nella Raccolta calogeriana (sottolineatura mia): «Un non so che Danielle da Luca ha composta una Poetica volgare per uso della Lingua volgare [...]. D. Giovanni Britaro m'ha pregato ch'io la vegga, il che ho fatto volentieri, perché novamente la Poetica d'Aristotele et d'Horatio haveva veduta. Che volete più! Ho acquistato il nome di pacientia appo il Melano, che senza dispensarmi son leggendo pervenuto al fine: brievemente più errori v'ha che sillabe, errori dico, che non ricevono scusa alcuna. Che faremo M. Filippo il Bembo non sa di questa lingua se non quanto ne sa, cioè poca al mio giuditio, né con quella poca scientia la può ajutar punto, che ad altra opera intende. Il Trifone nel quale speravano tanto gli buomini, nulla ne sa, e gli altri men che nulla. A voi toccherebbe per questa impresa. Ma che dico io? Voi sete servitore altrui, ne di questa servitù uscirete forse mai, et ciò richiederebbe la libertà di tre o quattro anni. Così a me pare che più tosto possiamo piangere la morente lingua nostra, che ajutarla» (CALOGERÀ, Raccolta d'opuscoli, vol. XLVII, I752, pp. 425 -26). La lettera, per il riferimento alla poetica del Daniello, sarebbe databile al i 536 , se l'editore settecentesco non dicesse che è del I 537 . Siamo in ogni modo alla stessa altezza cronologica dei sonetti.

I6. Per i testi letterari del Valentini conservati si veda l'introduzione della Felici a VaLENTINI, Il principe fanciullo, p. 3 I nota 90, e Roncaccia, Un frammento critico sulle Rime del Bembo cit., pp. 707 nota 3.

17. C. Dionisotti, Fortuna del Petrarca nel Quattrocento, «Italia medioevale e umanistica», 17 (1974), pp. 6I-II3, p. I09.

I8. Il componimento si trova in un codice miscellaneo conservato presso la Biblioteca Universitaria di Breslavia (cfr. P.O. Kristeller, Iter italicum, IV, London-Leiden, 1989, pp. 437-38), sotto la segnatura Milich Collection IV i 8 e precisamente alle cc. 83 r-89r, senza indubitabilmente proseguire, come erroneamente indicato dalla Felici, nelle cc. 89v-9ov. Nell'attesa di un'edizione integrale della canzone, le parti pubblicate dalla studiosa (VALENTINI, Il principe fanciullo, pp. 93-95), che parla più genericamente di «ode» (p. 3 I ) o di «lungo carme» (p. 93), vanno considerate con cautela, giacché la segmentazione attuata, non tenendo conto della costruzione metrica, non garantisce quella logica.

19. Cavazzuti, Ludovico Castelvetro cit., p. 196.

20. Come scrisse Contini, nei famosi Preliminari sulla lingua del Petrarca: «È un fatto che noi moderni ci sentiamo più solidali col temperamento, dico il temperamento linguistico, di Dante; ma è altrettanto un fatto che la sostanza della nostra tradizione è più prossima alla cultura petrarchesca» (Francesco Petrarca, Canzoniere, Torino, Einaudi, 1964, p. viiI). In 


\section{Ludovico Castelvetro e Filippo Valentini}

altri termini, come più recentemente sintetizza Santagata, a partire proprio dall'indicazione continiana che determinati settori del Canzoniere passino «attraverso Dante» (p. xxviri): «Dopo secoli di petrarchismo lirico solo la ricostruzione storica consente di vedere quanto sperimentalismo sottostia a scelte che diventeranno canoniche» (Francesco Petrarca, Canzoniere, a c. di M. Santagata, Milano, Mondadori, I996, p. xxxiv). Bisognerebbe allora tener conto del fatto che per figure come il Castelvetro o il Valentini, nonché per lo stesso Bembo, tale componente sperimentale della poesia del Petrarca doveva esser ancora molto evidente, se non costituire uno degli elementi chiave del confronto con il modello.

2 I. L'immagine, originata dal Salmo 84 (83), 7, deve come è noto il suo successo alla medievale composizione monastica Salve, Regina, già famosa al tempo di Dante (cfr. Purg. 7 , $82)$.

22. Contini, Preliminari sulla lingua del Petrarca cit., p. xxviı.

23. Contini notava appunto come tale tipo di enjambement potesse esser in Petrarca «fomentato dal troppo breve settenario» e come propriamente cinquecentesco fosse «il trasferimento sistematico di questa trovata nel sonetto» (Preliminari sulla lingua del Petrarca cit., p. XXIX).

24. Si può considerare come l'opera di selezione linguistica e di codificazione stilistica attuata dal Petrarca, oltre a determinare molto del futuro linguistico-letterario italiano, determinò anche le essenziali categorie di percezione del passato, del quale venivano così autorizzati (o meno) certi esiti di lingua e di stile. In questo caso specifico si può dire che l'esempio petrarchesco fa premio sulla fonte dantesca di Purg. 33, 33 «sì che non parli più com'om che sogna».

25. La rima, a differenza di quanto potrebbe sembrare a prima vista, non costituisce una ripetizione del sostantivo valle, proprio per la diversa natura avverbiale dell'espressione completa a valle.

26. Pietro Bembo, Prose e Rime, a c. di C. Dionisotti, Torino, Utet, I966², p. I 46.

27. Ludovico Castelvetro, Le Rime del Petrarca brevemente sposte, Basilea, Pietro de Sedabonis, i 582 , vol. II, p. I 2 e p. I68.

28. In un'altra lettera al Valentini pubblicata dal Calogerà, sempre del I 537 secondo la fonte, Castelvetro inserisce il Bembo tra i maggiori poeti del proprio tempo: «Bembo, Sannazzaro, Molza, Navagero, Vida, Fracastoro, et altri sono migliori poeti, che non sono questi Filelfo, Guerrino, con quella scuola di nessun valore» (CALOGERÀ, Raccolta d'opuscoli, vol. XLVII, I752, p. 426).

29. Bемвo, Prose e Rime, p. 626. La possibilità di una storia interna degli usi linguistici bembiani è stata, inoltre, recentemente indagata per l'Epistolario volgare da Massimo Prada, che ne mette in luce le escursioni diafasiche dovute sia a momenti compositivi più antichi, sia a diversi destinatari, così da indicare la complessità «della relazione che il Veneziano intravedeva tra testo letterario ed espressione comune», M. Prada, La lingua dell'Epistolario volgare di Pietro Bembo, Genova, Name, 2000, p. 247.

30. Come osserva Gorni, la canzone bembiana è di solito «una prova faticosa e squilibrata», in cui però Alma cortese può esser definita come «caso a parte» (Poeti del Cinquecento, a c. di G. Gorni, M. Danzi e S. Longhi, Milano-Napoli, Ricciardi, 200I, p. 4I).

31. Tale eco, più che dalla chiusa «mondo errante», già dantesca e petrarchesca, è realizzata attraverso il nesso logico che la introduce: Alma cortese, che dal mondo errante è l'incipit 


\section{Alberto Roncaccia}

bembiano che si può confrontare con i due incipit modenesi Spirti ben nati che del mondo errante e $O$ voi beati che del mondo errante. Per questi sonetti modenesi, si veda il già citato saggio della Felici, che ne trascrive sei dall'unico testimone, il codice Riccardiano 2835 , cc.65r-68v (VAlentini, Il principe fanciullo, pp. 32-33).

32. L'importanza storico-letteraria di tale edizione è ribadita da Gorni, anche editorialmente, sulla base di un'osservazione che fu già di Dionisotti (Poeti del Cinquecento cit., p. 45). 